\title{
A cross-sectional analysis of light at night, neighborhood sociodemographics and urinary 6-sulfatoxymelatonin concentrations: implications for the conduct of health studies
}

\author{
Susan Hurley ${ }^{1 *}$, David O Nelson ${ }^{1}$, Erika Garcia ${ }^{1}$, Robert Gunier ${ }^{1}$, Andrew Hertz ${ }^{1}$ and Peggy Reynolds ${ }^{1,2}$
}

\begin{abstract}
Background: There is accumulating evidence that circadian disruption, mediated by alterations in melatonin levels, may play an etiologic role in a wide variety of diseases. The degree to which light-at-night (LAN) and other factors can alter melatonin levels is not well-documented. Our primary objective was to evaluate the degree to which estimates of outdoor environmental LAN predict 6-sulftoxymelatonin (aMT6s), the primary urinary metabolite of melatonin. We also evaluated other potential behavioral, sociodemographic, and anthropomorphic predictors of aMT6s.
\end{abstract}

Methods: Study participants consisted of 303 members of the California Teachers Study who provided a 24-hour urine specimen and completed a self-administered questionnaire in 2000. Urinary aMT6s was measured using the Bühlmann ELISA. Outdoor LAN levels were estimated from satellite imagery data obtained from the U.S. Defense Meteorological Satellite Program's (DMSP) Operational Linescan System and assigned to study participants' geocoded residential address. Information on other potential predictors of aMT6s was derived from selfadministered surveys. Neighborhood socioeconomic status (SES) was based on U.S. Census block group data.

Results: Lower aMT6s levels were significantly associated with older age, shorter nights, and residential locations in lower SES neighborhoods. Outdoor sources of LAN estimated using low-dynamic range DMSP data had insufficient variability across urban neighborhoods to evaluate. While high-dynamic range DMSP offered much better variability, it was not significantly associated with urinary aMT6s.

Conclusions: Future health studies should utilize the high-dynamic range DMSP data and should consider other potential sources of circadian disruption associated with living in lower SES neighborhoods.

Keywords: Circadian disruption, Light at night, Melatonin, aMT6s, Socioeconomic status, Women

\section{Background}

There is growing evidence that environmental light pollution may play an etiologic role in a variety of diseases, including depression, cardiovascular disease, and cancer [1-10]. Such health effects are largely thought to be mediated via circadian disruption driven by alterations in melatonin production and secretion $[11,12]$. Melatonin, an endogenous hormone produced by the pineal gland,

\footnotetext{
* Correspondence: susan.hurley@cpic.org

'Cancer Prevention Institute of California, 2001 Center Street, Suite 700, Berkeley, CA 94704, USA

Full list of author information is available at the end of the article
}

is the primary hormonal modulator of circadian regulation in mammals and is strongly influenced by exposure to visible light, with levels peaking during the darkness of night [13]. Predictors of melatonin levels in humans generally have not yet been well-characterized [11,14-18]. While there is fairly strong evidence that indoor artificial light-at-night (LAN) is sufficient to suppress melatonin levels in humans, it is not known whether outdoor environmental light pollution is sufficient to exert a similar effect $[1,3,19]$. Fully understanding such predictors is a necessary prerequisite for implementing epidemiologic studies on this topic. 
The primary objective of the current analyses was to evaluate the degree to which estimates of outdoor environmental light-at-night (LAN) predict levels of 6-sulfatoxymelatonin (aMT6s), the primary urinary metabolite of melatonin, among 303 California women. Secondarily, we evaluated whether selected anthropomorphic characteristics and behavioral and sociodemographic factors predict urinary concentrations of aMT6s.

\section{Results}

Creatinine adjusted urinary aMT6s concentrations ranged from $0.58 \mathrm{ng} / \mathrm{mg}$-creatinine to $102.06 \mathrm{ng} / \mathrm{mg}$-creatinine with a mean of $20.66 \mathrm{ng} / \mathrm{mg}$-creatinine, a standard deviation of $17.78 \mathrm{ng} / \mathrm{mg}$-creatinine and an interquartile range of $17.74 \mathrm{ng} / \mathrm{mg}$-creatinine.

The characteristics of the study population are summarized in Table 1. Similar to the entire CTS cohort, the study population is predominantly non-Hispanic white $(85 \%)$ and middle-aged (mean age $=55$ years). Despite arising from an occupational cohort that requires at least a 4-year college degree among its members, study participants lived in both high and low SES neighborhoods, although skewed towards higher SES neighborhoods. By design, study participants were oversampled from rural neighborhoods $(60.4 \%)$.

Table 2 summarizes estimates of outdoor LAN exposures. The average length-of-night was 11 hours, reflecting the fact that most urine specimens were collected in the winter and early spring months. The radiance estimates of outdoor LAN ranged from 4 to 63 , with a mean value of 43.4 , for the low-dynamic range 2000 data, and 3.8 to 465.2 , with a mean of 128.4 , for the high-dynamic range 2006 data. Figure 1 illustrates the greater range and variability in radiance estimates offered by the high-dynamic range data. More importantly, Figure 1 illustrates that the low-dynamic range data lacks sufficient variability at the upper range to discriminate areas with higher LAN values within urban neighborhoods. In addition, LAN values were universally higher among women living in urban compared to rural neighborhoods, with no overlap in values across rural and urban neighborhoods. In contrast, the high-dynamic range data offers variability in values within both rural and urban areas as well as some overlap in values across urban and rural areas. Consequently, we chose to rely on the high-dynamic range data for the remainder of our analyses.

The results from our random forests analyses to identify the most important predictors of urinary aMT6s concentrations, based on the scaled variable importance values (mean divided by the standard deviation), identified the following 8 variables as potentially important predictors of aMT6s: hormone therapy use; oral contraceptive use; length-of-night; coffee consumption; strenuous exercise in the past 3 years; age; menopausal status; and neighborhood SES. Overall, however, none of these variables, individually or combined, explained a substantial proportion of the variability in measured aMT6s levels (data not shown).

Prior to conducting our regression analyses, we examined the shape of the relationship between SES and aMT6s. A plot of the smoothed aMT6s on the first component of our SES PCA (Figure 2) demonstrates that increases in melatonin are seen with increases in SES but only among those living in neighborhoods for which the PCA value for SES did not exceed zero. Once the summary neighborhood SES reaches the average value (represented by zero), the relationship flattens out. Consequently, in our regression model selection process, we represented the summary SES measure as a continuous, piecewise linear function that has one join point at zero and is constrained to have zero slope when the PCA value exceeds zero.

Model selection by backwards elimination, starting with the 8 variables identified through our random forests analyses (see above), identified length-of-night, SES, and age as the only significant predictors of aMT6s (Table 3). Levels of aMT6s declined with older age and increased with longer nights and higher SES. Among the subset of women over the age of 55 years, SES was not a significant predictor of aMT6s while length-of-night and age became stronger and more significant predictors. Overall, these models explained very little of the variance in aMT6s $\left(R^{2}\right.$ for all women $=0.03 ; R^{2}$ for women $>55$ years $\left.=0.07\right)$.

Although the outdoor LAN variable was not selected through our stepwise model building approach, because it was of primary interest to us, we evaluated it separately in age-adjusted regression models and found it to have a very small inverse association with aMT6s that was not significant in either the full study population $(\beta=-0.0028$, se $=0.008, \mathrm{p}=0.73)$ or among those over age $55(\beta=-0.0062$, se $=0.012, \mathrm{p}=0.62)$.

\section{Discussion}

Overall, results from our study failed to identify factors that substantially predict urinary measures of aMT6s. The only factors that were significantly related to aMT6s levels were age, length-of-night, and SES and together they explained very little of the variance in aMT6s levels. Nevertheless, our study does provide some important findings relevant to future investigations of health outcomes related to melatonin and/or LAN exposures.

There is increasing interest in the use of satellite imagery data to evaluate potential effects of outdoor environmental light pollution in wildlife and in human populations, including a number of studies that have investigated potential links to cancer incidence [20-22]. Our analyses demonstrated that the widely-available low-dynamic range annual satellite imagery data are 
Table 1 Characteristics of study population $(n=303)$

Characteristics Distribution

Age at urine collection, years (mean,standard deviation)

BMl at urine collection, $\mathrm{kg} / \mathrm{m}^{2}$ (mean,standard deviation)

Height at CTS baseline survey, inches (mean,standard deviation)

$55.0(11.9)$

$26.6(5.8)$

$65.0(2.7)$

Packyears of smoking (mean,standard deviation)

$4.0(10.0)$

Pillyears of aspirin use (mean,standard deviation)

$0.69(2.0)$

Coffee consumption, average grams/day

$238.2(184.7)$

(mean,standard deviation)

Coffee consumption, average times/day (mean,standard deviation)

$0.97(0.72)$

Strenuous exercise, 3-years prior to baseline, hours/wk (mean,standard deviation)

$1.6(2.4)$

Strenuous exercise, lifetime prior to baseline, hours/wk (mean,standard deviation)

Age at first full-term pregnancy, years (mean,standard deviation)

Parity, total number of live and still births (mean,standard deviation)

\begin{tabular}{ll}
\hline Race/ethnicity (n,\%) & $258(85.1)$ \\
Non-Hispanic white & $18(5.9)$ \\
Hispanic & $3(1.0)$ \\
Black & $13(4.3)$ \\
Asian \& Pacific Islander & $11(3.6)$ \\
Other/unknown & $131(43.2)$ \\
\hline Menopausal Status, at baseline(n,\%) & $147(48.5)$ \\
Postmenospausal & $25(8.3)$ \\
Premenopausal or Perimenopausal & \\
Unknown & $86(28.4)$ \\
\hline Alcohol Consumption, year prior to baseline (n, \%) \\
None & $176(58.1)$ \\
$<20$ g/day & $27(8.9)$ \\
$\geq 20$ g/day & $14(4.6)$ \\
Unknown & \\
\hline Tobacco Smoke Exposure, at baseline (n, \%) & $61(20.1)$ \\
None & $142(46.9)$ \\
Passive only & $86(28.4)$ \\
Former active smoker & $12(4.0)$ \\
Current active smoker & $2(0.7)$ \\
Unknown &
\end{tabular}

Oral contraceptive use, at baseline $(n, \%)$

$\begin{array}{ll}\text { Never } & 81(26.7) \\ \text { Former } & 198(65.4) \\ \text { Current } & 16(5.3) \\ \text { Unknown } & 8(2.6)\end{array}$

Antidepressant use, daily use for at least 2 months at baseline
Table 1 Characteristics of study population $(n=303)$ (Continued)

\begin{tabular}{|c|c|}
\hline Yes & $29(9.6)$ \\
\hline No & $237(78.2)$ \\
\hline Unknown & $37(12.2)$ \\
\hline \multicolumn{2}{|l|}{ Hormone therapy use, at baseline $(n, \%)$} \\
\hline Premenopausal & $142(46.9)$ \\
\hline Peri/post-menopausal, never used & $34(11.22)$ \\
\hline Peri/post-menopausal, prior use & $16(5.3)$ \\
\hline Peri/post-menopasual, current use & $71(23.4)$ \\
\hline Other/unknown & $40(13.2)$ \\
\hline \multicolumn{2}{|l|}{ Regular aspirin use, years at baseline } \\
\hline None & $240(79.2)$ \\
\hline$<1$ & $11(3.6)$ \\
\hline 1 & $2(0.7)$ \\
\hline 2 & $4(1.3)$ \\
\hline $3-4$ & $7(2.3)$ \\
\hline $5-9$ & $6(2.0)$ \\
\hline $10+$ & $31(10.2)$ \\
\hline Unknown/missing & $2(0.7)$ \\
\hline \multicolumn{2}{|l|}{ Regular aspirin use, days/week at baseline } \\
\hline None & $240(79.2)$ \\
\hline $1-3$ & $41(13.5)$ \\
\hline $4-6$ & $9(3.0)$ \\
\hline 7 & $12(4.0)$ \\
\hline Unknown & $1(0.3)$ \\
\hline \multicolumn{2}{|l|}{ Daily Use of Calcium Blockers, in 2000} \\
\hline Yes & $4(1.3)$ \\
\hline No & $246(81.2)$ \\
\hline Unknown & $53(17.5)$ \\
\hline \multicolumn{2}{|l|}{ Neighborhood Urbanization } \\
\hline Urban & $120(39.6)$ \\
\hline Rural & $183(60.4)$ \\
\hline \multicolumn{2}{|l|}{ Hours of sleep/night } \\
\hline$<5$ hours & $3(1.0)$ \\
\hline 5-6 hours & $63(20.8)$ \\
\hline 7-9 hours & $226(74.6)$ \\
\hline $10+$ hours & $4(1.3)$ \\
\hline Unknown & $7(2.3)$ \\
\hline \multicolumn{2}{|l|}{$\begin{array}{l}\text { Neighborhood Socioeconomic Status } \\
\text { (mean, range) }\end{array}$} \\
\hline Median Family Income (\$) & $\begin{array}{l}76,782(37,337- \\
140,387)\end{array}$ \\
\hline Percent of population below poverty level & $6.8(0.0-37.6)$ \\
\hline Percent of adults age $25+$ with college degree & $34.3(1.5-79.0)$ \\
\hline Percent of adults without a high school degree & $14.6(1.2-56.8)$ \\
\hline $\begin{array}{l}\text { Percent of adults employed in a professional } \\
\text { occupation }\end{array}$ & $44.3(6.7-74.3)$ \\
\hline
\end{tabular}


Table 2 Estimates of outdoor LAN among study participants $(n=303)$

\begin{tabular}{ll}
\hline & Mean (Range) \\
\hline $\begin{array}{l}\text { Outdoor LAN satellite values } \\
\text { (annual average radiance) }\end{array}$ & \\
$\quad$ Low-dynamic range, 2000 average (DN units) & $43.4(4-63)$ \\
$\begin{array}{l}\text { High-dynamic range, } 2006 \text { average } \\
\text { (scaled radiance units) }\end{array}$ & $128.4(3.8-465.2)$ \\
\hline Length-of-night on day of urine collection (hours) & $11(9.2-14.4)$ \\
\hline
\end{tabular}

insufficient for distinguishing areas with differing LAN values within suburban and urban areas. Since many health outcomes, including many cancers, are more common in urbanized areas [23-27], use of the low-dynamic range satellite data will likely be inadequate for investigating LAN exposures within such areas. While the highdynamic range data are currently only available for 2006, efforts are underway to develop such high-dynamic range data for other years [28]. Researchers interested in this topic would be prudent to pursue such data.

The other novel finding generated by our analyses was the significance of neighborhood SES. Our analysis showed that aMT6s concentrations tended to be low among women living in the lowest SES areas and increased with greater neighborhood SES up to a point after which the concentration leveled off and possibly declined slightly at the very highest levels of SES. Furthermore, this relationship, while statistically significant in the full study population, appeared to be of lesser importance in the women over age 55 . Overall, very little is known about how melatonin varies by SES. Similar to our findings, a study of approximately 200 Canadian women reported that urinary measures of aMT6s were lower among the lowest educated women (i.e. high school diploma or less), highest in the middle category of education, with a slight decrease in the highest educated group [29]. In contrast, Burgess and colleagues in their evaluation of predictors of salivary measures of melatonin found education was not a significant predictor [30].

Interpretation of our findings on SES can only be speculative. It is possible that the neighborhood measure of SES is serving as a proxy for a number of behavioral and/or anthropomorphic factors that influence melatonin levels. We did, however, incorporate variables in our model selection process for many of the most important of these factors including alcohol consumption, smoking, BMI, use of exogenous hormones and other medications, coffee consumption, and physical activity none of which were independent and significant predictors of aMT6s. Another possibility is that low SES neighborhoods may have environmental stressors (e.g., noise, crime) other than light pollution that we did not measure in our study but could disrupt circadian rhythms and lead to lower levels of aMT6s. Given that a number of the health outcomes of high interest with respect to circadian disruption display strong SES-related risk gradients, research into other features of the built environment represents an important area for future inquiry.

In addition to SES, age and length-of-night were the only other significant predictors of aMT6s identified in our analysis. These findings are generally consistent with the limited body of literature on this topic. Two studies have reported seasonal differences in melatonin levels with higher levels associated with greater length-of-night [15,17] and lower levels in the summer months [17], although two other studies reported no relationship with month [18] or season [31]. Most studies have reported declines in melatonin levels with increasing age, albeit most have reported this relationship to be linear $[15,17,18,31-33]$. Consistent with our results, two studies noted more dramatic age-related declines among older individuals [34,35]; conversely, a few other studies suggested that the age-related declines in melatonin are greater, or are limited to, early adulthood and then leveloff [18,36-38]. Measurements of melatonin in older adults living under controlled light-dark conditions in one study reported no age-related declines in melatonin levels, leading the authors to suggest that changes in sleep behaviors might be responsible for the age-related declines reported in other observational studies [39]. In our study, however, we observed no differences in sleep

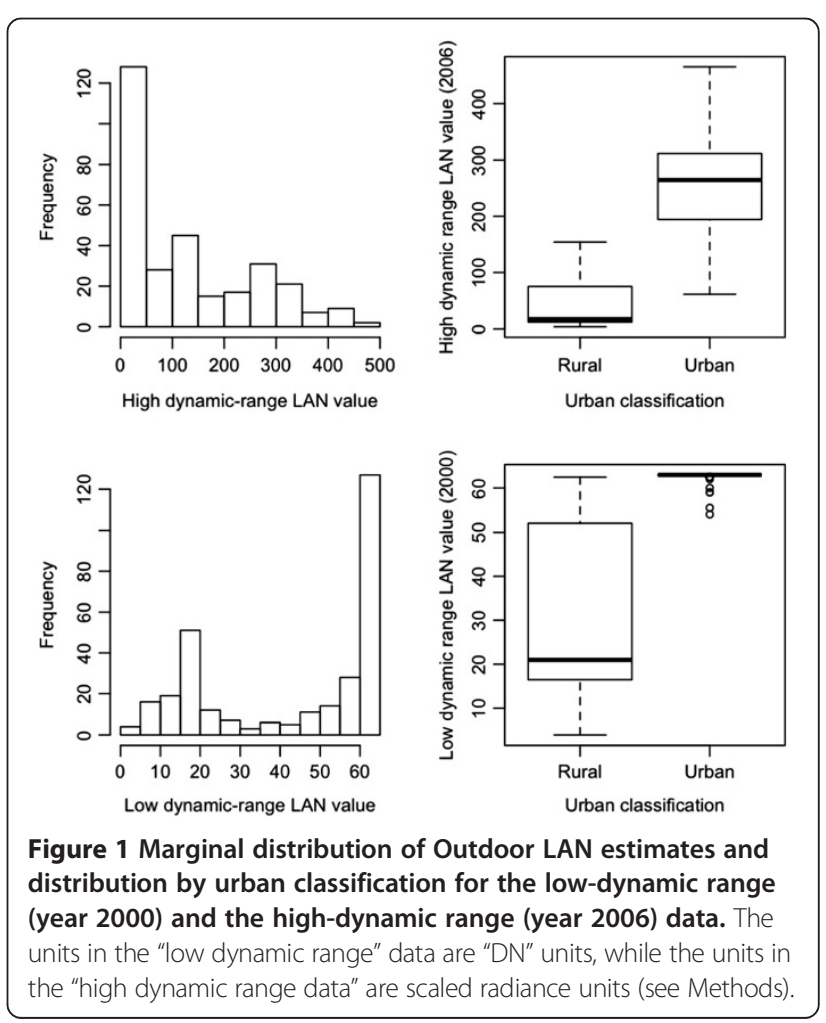




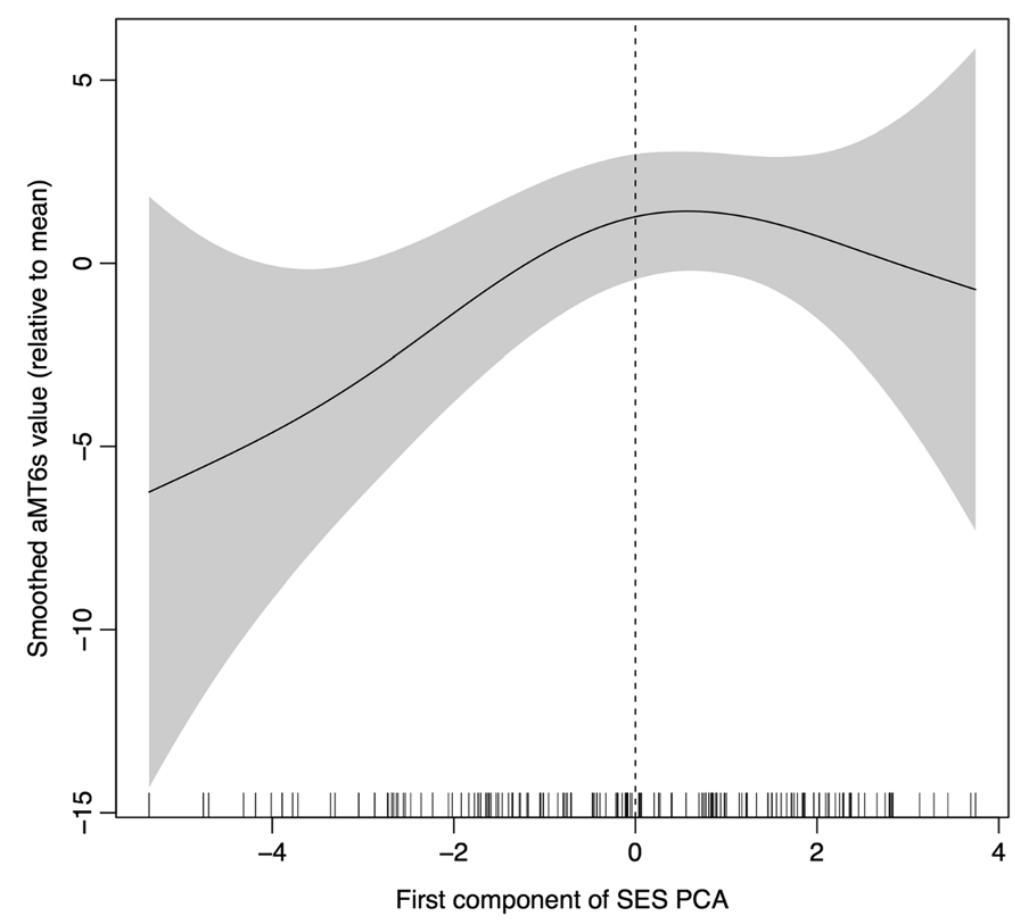

Figure 2 Estimated relationship between neighborhood SES and aMT6s, along with the pointwise $95 \%$ confidence band (shaded in gray). The relationship increases until SES equals zero, at which point it flattens out and no statistically significant slope remains (the dashed vertical line indicated where the SES PCA component is equal to zero). The lines across the bottom indicate the SES values for the sample. Note that the spline only shows deviation of aMT6s relative to the average aMT6s value across the sample.

duration by age group (data not show). Our results, in the context of the somewhat conflicting literature on this, underscore the need to carefully consider the shape of the age-related risks when conducting health-related research on this topic. The fact that we observed a shift in the relationship between age and aMT6s around age 55 suggests this may be especially important when evaluating health risks in women, whose risks often change in their early- to mid-50s after menopause. This may be particularly relevant to breast cancer which exhibits a different constellation of risk relationships in pre- and postmenopausal women and for which LAN exposures have been postulated to be a potential risk factor.

Our evaluation of outdoor LAN, which showed a very modest inverse, but not statistically significant, relationship to aMT6s concentrations, was hindered by a number of obstacles. While we used the best satellite imagery data available to objectively estimate outdoor LAN values, because the high-dynamic range data was only available for 2006, the LAN estimates were not temporally congruent with the urine in which the aMT6s was measured (collected in 2000). An examination of the low-dynamic range data for all years spanning this time period (2000-2006), however, suggested relative stability in LAN values over this time for the study area. Furthermore, the Spearman rank correlation between the 2000 low-dynamic range data and the 2006 high-dynamic range data was 0.96 .

Another reason for the lack of an association seen between outdoor LAN and urinary measures of aMT6s could be that while the satellite imagery data may be an adequate predictor of outdoor ambient light, it may not reflect light exposures experienced at night when participants are likely to spend the majority of their time

Table 3 Regression coefficients $(\beta)$ for final model estimating $\log _{2}$ creatinine-adjusted aMT6s

\begin{tabular}{llrr}
\hline & Variable & $\boldsymbol{\beta}$, standard error & t statistic (2-sided $\mathbf{p}$-value) \\
\hline All Subjects & Length-of-night & $0.1081,0.0612$ & $1.76(0.08)$ \\
& Age & $-0.0113,0.0066$ & $-1.79(0.09)$ \\
& SES & $0.1375,0.0637$ & $2.16(0.03)$ \\
\hline Ages $>55$ years & Length-of-night & $0.2341,0.0963$ & $2.43(0.02)$ \\
& Age & $-0.0284,0.0152$ & $-1.87(0.06)$ \\
\hline
\end{tabular}


indoors. Intervening factors, such as the use of blinds/ curtains, time spent indoors, other sources of indoor light, etc. all are likely contributors to LAN exposures. The importance of this is underscored by findings from a recent study that compared estimates of LAN from satellite imagery data to calibrated photometric measurements obtained from personal monitors and reported that satellite imagery data did not correlate with personal photometric measurements [40]. Thus, it is important that studies aimed at evaluating outdoor LAN exposures do so with full consideration of these other factors.

Finally, there are a number of limitations in the estimates of our melatonin levels that are worth noting. The urine specimens were collected over a 24-hour time period. This precludes our ability to examine the timing of peak melatonin concentration which is likely an important factor in circadian disruption [13]. Furthermore, while there is a good deal of evidence that first morning urine captures peak night-time melatonin excretion, the degree to which 24-hour urine specimens reflect this is not well-documented [13,31]. The aMT6s assay, however, is a well-validated biomarker, demonstrating good correlation with serum melatonin levels [13], and sufficient stability over time $[13,15,31,41,42]$ to serve as a reasonable estimate of chronic levels.

\section{Conclusions}

There is growing evidence that circadian disruption, mediated by alterations in melatonin secretion driven by night-time light exposures may play an etiologic role in a large array of diseases. The use of satellite imagery data to estimate ambient measures of LAN offers an innovative and inexpensive source of data to test emerging hypotheses on this topic. The results from our analyses highlight some important cautionary limitations to the use of such data as well as point to some additional avenues of pursuit, including the elucidation of factors associated with neighborhood SES that may play a role in circadian disruption.

\section{Methods}

\section{Study participants}

Study participants consisted of 303 members of the California Teachers Study (CTS) residing in the San Francisco Bay Area, who as part of a special substudy conducted in 2000, completed a self-administered questionnaire, an in-person interview, and supplied a 24hour urine sample. The CTS is a prospective cohort study initiated in 1995, consisting of 133,479 female professional California school employees for whom extensive information has been collected through a baseline questionnaire and three subsequent mailed questionnaires [43]. Members of the special substudy included a random sample of 528 CTS participants who were $\leq 85$ years old when the cohort was established in 1995 , and who resided in the substudy area (comprised of western Alameda, Santa Clara, San Mateo, Santa Cruz, Monterey, and northern San Benito counties). Forty-four (8\%) of these women were not contacted because they had died, moved out of the substudy area, or could not be located. Of the 484 women invited to participate, 328 agreed, of whom 303 provided a 24-hour urine sample. Further details of the special substudy can be found elsewhere [44]. The use of human subjects was reviewed and approved by the California Health and Human Services Agency, committee for the Protection of Human Subjects and the Cancer Prevention Institute of California's Institutional Review Board.

\section{Data and specimen collection Specimen collection}

Participants were given urine collection kits and instructed to collect all urine produced over the next 24-hour period. Participants were asked to use cold packs to keep the sample chilled and, if willing, to refrigerate it during the 24-hour collection period. After 24-hours, staff collected and stored the samples at $-20^{\circ}$ Celsius until they could be delivered to our laboratory (within 2 weeks) where it was thawed, aliquoted into $10 \mathrm{~mL}$ vials, and frozen at $-70^{\circ}$ Celsius. Prior to aliquoting, total urine volume was measured and recorded.

\section{Laboratory methods}

Urinary concentration of aMT6s was measured by Pacific Toxicology Laboratory (Chatsworth, CA, USA) using Bühlmann aMT6s enzyme-linked immunosorbent assay (ELISA) kits purchased through ALPCO (Salem, NH, USA). The aMT6s ELISA is a competitive immunoassay using a capture antibody technique that has a lower detection limit of $0.8 \mathrm{ng} / \mathrm{mL}$ and an analytic high sensitivity limit of $40 \mathrm{ng} / \mathrm{mL}$. The functional sensitivity of the assay was $1.5 \mathrm{ng} / \mathrm{mL}$, while the intra-and interassay precision were $7.1 \%$ and $11.9 \%$, respectively. Urinary creatinine levels were obtained from laboratory assays using a modified-rate Jaffe method conducted as part of a prior analysis by the University of Alabama, Birmingham in 2000. aMT6s levels were creatinineadjusted by dividing the aMT6s concentration by the creatinine concentration.

Four samples were below the detection limit and five samples exceeded the analytic high sensitivity limit (3\% of samples total). Using PROC MI with a TRANSFORM LOG(var) statement in SAS 9.3 we imputed the values below and above the limit of detection, setting range parameters of $0-0.8 \mathrm{ng} / \mathrm{mL}$ and $40-60 \mathrm{ng} / \mathrm{mL}$ respectively. The upper range of $60 \mathrm{ng} / \mathrm{mL}$ for those samples above the analytic high sensitivity limit was based upon upper levels observed in 24-hour samples from women in a 
previous study [18]. We used the first imputation to assign values of aMT6s to these nine participants.

\section{Outdoor environmental LAN data}

Information on outdoor exposures to ambient LAN was derived from nighttime satellite imagery data obtained from the U.S. Defense Meteorological Satellite Program's Operational Linescan System (DMSP-OLS) [45]. This database contains annual composites, made after excluding the outer quarters of the satellite swath, light from the sun and moon, glare, clouds, and atmospheric lightning. Ephemeral events, such as fires, are also discarded. While these images capture only a fraction of the light originating from the earth's surface, they accurately represent the relative levels of nighttime illumination at ground level [45]. The imagery data is georectified to a 30 arc-second grid (equivalent to approximately one-square kilometer). Two kinds of radiance information were available: adaptive gain, low dynamic range data; and fixed-gain, high dynamic range data. The low dynamic range data are available for multiple years, but consist of unit-free (called DN, or "Digital Number" units) data with a dynamic range of six bits, i.e., integers ranging from 0 (background noise) to 63 . The fixed gain, high dynamic range data, is available only for 2006 and is obtained by combining information from several fixed-gain sensors. It consists of floating point numbers ("scaled radiance" units) that, if needed, can be scaled by $1.51586 \times 10^{-10}$ to obtain data that are watts $/ \mathrm{sr} / \mathrm{cm}^{2}$ (see the F16_2006_radiance_readme.txt file in reference [45] for caveats with respect to this scale factor). Such scaling was not performed for these analyses. Residences from study participants were assigned the average nighttime radiance value for the grid cell in which they were located using spatial analysis tools available in the raster, rgdal, and sp packages within $\mathrm{R}$ [46]. Initially we assigned to residences both the low-dynamic range data for 2000 and the high-dynamic range data for 2006.

As an additional measure of LAN we generated a "length-of-night" variable, created by linking the date of urine collection with daily sunrise and sunset data for San Francisco for the year 2000 obtained from the Naval Observatory [47]. From these data we calculated the hours of darkness on the day of urine collection.

\section{Anthropomorphic and behavioral factors}

Information on anthropomorphic and behavioral factors of interest was derived from the CTS surveys. Factors were considered as potential predictors for these analyses based on a review of the limited literature on this topic [15-18,31-35,37,38,42,48-51] and included: age, race/ethnicity, body mass index (BMI), height, parity, number of live births, age at first full-term pregnancy, pack-years of smoking, alcohol consumption, oral contraceptive use, hormone therapy use, menopausal status, strenuous physical activity, average hours of sleep per night, age at menarche, coffee consumption, and the use of aspirin, calcium blockers and antidepressants.

\section{Sociodemographic data}

Information on neighborhood socioeconomic characteristics and urbanization were derived from U.S. Census 2000 data. We used the ArcGIS, version 9.2 (ESRI, Redlands, CA, USA) to geocode the participants' home addresses to both a latitude/longitude and to a Census 2000 block group. Data on socioeconomic status (SES) included: percentage of adults over age 25 years having completed a college degree or higher; percentage of adults without a high school degree; income (median family income), occupation (percentage of adults employed in managerial/professional occupations), and poverty (percentage of population below the poverty line). The degree of neighborhood urbanization was characterized as either urban/suburban or rural, based on a previously-developed algorithm using a combination of census block group characteristics, details of which can be found elsewhere [26].

\section{Statistical methods}

Given the extensive information collected on potential predictors of melatonin levels, our initial approach was to use Breiman's random-forest-based (RF) variable importance measures, as described by Lunetta [52] to identify the most important predictors of $\log _{2}$ transformed, creatinine adjusted aMT6s from the large number of factors for which we had information. (The aMT6s levels were highly skewed to the right, necessitating the $\log _{2}$ transformation.) This approach allowed us to compose a list of candidate variables, ordered by their relative importance, taking into account both multiple testing and potential interactions. The top ranking variables were then considered in linear models to further quantify predictors of aMT6s concentrations. Final model selection via R's stepAIC function was chosen in a backwards elimination process using the Akaike information criterion (AIC) to quantify the model fit.

We first focused on determining the most appropriate model specification for age. A plot, obtained by fitting a Generalized Additive Model (GAM) with an intercept and a spline term for age, demonstrated a decrease in aMT6s with increasing age, with a possible downward curvature at age 55. An analysis of variance to compare null, linear, and quadratic linear models for the regression of the creatinine adjusted aMT6s concentrations on age, however, indicated that the best fitting model was one with age modeled as a simple linear term. Because restricting our analyses to women over age 55 resulted in a much stronger and significant linear effect for age 
than observed in the full study population, we conducted our subsequent analyses on the full study sample, as well as among women over the age of 55 . To address the high degree of collinearity between the SES variables, we conducted a principal components analysis (PCA) to create a composite measure of SES based on the five individual components as described above. The loadings of the first principal component were then used as a composite measure in the random forests and regression models.

\section{Abbreviations}

aMT6s: 6-sulftoxymelatonin; LAN: Light at night; CTS: California Teachers Study; SES: Socioeconomic status; se: standard error.

\section{Competing interests}

The authors declare that they have no competing interests.

\section{Authors' contributions}

$\mathrm{SH}$ participated in the design and coordination of the study and drafted the manuscript. DON obtained the satellite imagery data, assisted with the development of the GIS-based measures of SES, and oversaw the statistical analysis. EG conducted QA/QC on the laboratory data and assisted in the data analysis. RG assisted in the development of the study design and coordinated the laboratory assays. AH geocoded the residential addresses and assisted with assigning GIS-based measures to residences. PR participated in the design of the study and helped draft the manuscript. All authors read and approved the final version of the manuscript.

\section{Acknowledgements}

We express our appreciation to all the participants in the California Teachers Study and to the researchers, analysts and staff who have contributed so much for the success of this research project. We also thank Christine Duffy for coordinating the urine collection and storage, Minhthu Le for administrative support, and the CTS Steering Committee members who are responsible for the formation and maintenance of the cohort within which this study was conducted but who did not directly contribute to the current paper: Leslie Bernstein, Hoda Anton-Culver, Jessica Clague, Christina A. Clarke, Dennis Deapen, Pam Horn-Ross, James V. Lacey Jr, Yani Lu, Huiyan Ma, Susan L. Neuhausen, Hannah Park, Rich Pinder, Fredrick Schumacher, Sophia S. Wang, and Argyrios Ziogas. This research and the development of this manuscript was supported by funding from the California Breast Cancer Research Program (Grant \#16|B-0071) and National Cancer Institute grants R01 CA77398 and K05 CA136967.

\section{Author details}

${ }^{1}$ Cancer Prevention Institute of California, 2001 Center Street, Suite 700, Berkeley, CA 94704, USA. ${ }^{2}$ Department of Health Research and Policy, Stanford University School of Medicine, Stanford, CA 94305, USA.

Received: 17 July 2013 Accepted: 26 August 2013

Published: 30 August 2013

\section{References}

1. Chepesiuk R: Missing the dark: health effects of light pollution. Environ Health Perspect 2009, 117:A20-A27.

2. Hede K: Cancer and the circadian clock: Has the time finally come? J Natl Cancer Inst 2009, 101:550-553.

3. Kantermann $T$, Roenneberg $\mathrm{T}$ : Is light-at-night a health risk factor or a health risk predictor? Chronobiol Int 2009, 26:1069-1074

4. Korkmaz A: Epigenetic actions of melatonin. J Pineal Res 2009, 46:117-118.

5. Roenneberg T, Lucas RJ: Light, endocrine systems, and cancer-a view from circadian biologists. Neuro Endocrinol Lett 2002, 23(Suppl 2):82-83.

6. Sahar S, Sassone-Corsi P: Metabolism and cancer: the circadian clock connection. Nat Rev Cancer 2009, 9:886-896.

7. Stevens RG: Circadian disruption and breast cancer: from melatonin to clock genes. Epidemiology 2005, 16:254-258.

8. Stevens RG: Working against our endogenous circadian clock: breast cancer and electric lighting in the modern world. Mutat Res 2009, 679:6-8.
9. Stevens RG: Electric light causes cancer? Surely you're joking, Mr. Stevens. Mutat Res 2009, 682:1-6.

10. Stevens RG: Light-at-night, circadian disruption and breast cancer: assessment of existing evidence. Int J Epidemiol 2009, 38:963-970.

11. Dopfel RP, Schulmeister K, Schernhammer ES: Nutritional and lifestyle correlates of the cancer-protective hormone melatonin. Cancer Detect Prev 2007, 31:140-148.

12. Schernhammer ES, Schulmeister K: Melatonin and cancer risk: does light at night compromise physiologic cancer protection by lowering serum melatonin levels? Br J Cancer 2004, 90:941-943.

13. Mirick DK, Davis S: Melatonin as a biomarker of circadian dysregulation. Cancer Epidemiol Biomarkers Prev 2008, 17:3306-3313.

14. Arendt J: Melatonin: characteristics, concerns, and prospects. J Biol Rhythms 2005, 20:291-303.

15. Davis S, Kaune WT, Mirick DK, Chen C, Stevens RG: Residential magnetic fields, light-at-night, and nocturnal urinary 6-sulfatoxymelatonin concentration in women. Am J Epidemiol 2001, 154:591-600.

16. Grundy A, Sanchez M, Richardson H, Tranmer J, Borugian M, Graham CH, Aronson KJ: Light intensity exposure, sleep duration, physical activity, and biomarkers of melatonin among rotating shift nurses. Chronobiol Int 2009, 26:1443-1461.

17. Knight JA, Thompson S, Raboud JM, Hoffman BR: Light and exercise and melatonin production in women. Am J Epidemiol 2005, 162:1114-1122.

18. Schernhammer ES, Kroenke CH, Dowsett M, Folkerd E, Hankinson SE: Urinary 6-sulfatoxymelatonin levels and their correlations with lifestyle factors and steroid hormone levels. J Pineal Res 2006, 40:116-124.

19. Graham C, Cook MR, Gerkovich MM, Sastre A: Examination of the melatonin hypothesis in women exposed at night to EMF or bright light. Environ Health Perspect 2001, 109:501-507.

20. Kloog I, Haim A, Stevens RG, Barchana M, Portnov BA: Light at night co-distributes with incident breast but not lung cancer in the female population of Israel. Chronobiol Int 2008, 25:65-81.

21. Kloog I, Haim A, Stevens RG, Portnov BA: Global co-distribution of Light At Night (Lan) and cancers of prostate, colon, and lung in men. Chronobiol Int 2009, 26:108-125.

22. Kloog I, Stevens RG, Haim A, Portnov BA: Nighttime light level co-distributes with breast cancer incidence worldwide. Cancer Causes Control 2010, 21:2059-2068.

23. Doll R: Urban and rural factors in the aetiology of cancer. Int I Cancer 1991, 47:803-810.

24. Haenszel W, Marcus SC, Zimmerer EG: Cancer morbidity in urban and rural lowa. Public Health Monogr 1956, 37:1-85.

25. Parkin DM, Bray F, Ferlay J, Pisani P: Global Cancer Statistics, 2002. Ca Cancer J Clin 2005, 55:74-108.

26. Reynolds P, Hurley S, Goldberg DE, Anton-Culver H, Bernstein L, Deapen D, Horn-Ross PL, Peel D, Pinder R, Ross RK, West D, Wright WE, Ziogas A: Regional variations in breast cancer among California teachers. Epidemiology 2004, 15:746-754

27. Reynolds P, Hurley SE, Quach AT, Rosen H, Von Behren J, Hertz A, Smith D: Regional variations in breast cancer incidence among California women, 1988-1997. Cancer Causes Control 2005, 16:139-150.

28. Ziskin DB, Hsu FC, Ghosh T, Elvidge C: Methods used for the 2006 radiance lights. In Proceedings Of The Asia Pacific Advanced Network Meeting 2010; 2010

29. Levallois P, Dumont M, Touitou Y, Gingras S, Masse B, Gauvin D, Kroger E, Bourdages M, Douville P: Effects of electric and magnetic fields from high-power lines on female urinary excretion of 6-Sulfatoxymelatonin. Am J Epidemiol 2001, 154:601-609.

30. Burgess HJ, Fogg LF: Individual differences in the amount and timing of salivary melatonin secretion. Plos One 2008, 3:E3055.

31. Travis RC, Allen DS, Fentiman IS, Key TJ: Melatonin and breast cancer: a prospective study. J Natl Cancer Inst 2004, 96:475-482.

32. Mahlberg R, Tilmann A, Salewski L, Kunz D: Normative data on the daily profile of urinary 6-sulfatoxymelatonin in healthy subjects between the ages of 20 and 84. Psychoneuroendocrinology 2006, 31:634-641.

33. Wetterberg L, Bratlid T, Von Knorring L, Eberhard G, Yuwiler A: A multinational study of the relationships between nighttime urinary melatonin production, age, gender, body size, and latitude. Eur Arch Psychiatry Clin Neurosci 1999, 249:256-262

34. Bojkowski CJ, Arendt J: Factors influencing urinary 6-sulphatoxymelatonin, a major melatonin metabolite, in normal human subjects. Clin Endocrinol (Oxf) 1990, 33:435-444. 
35. Youngstedt SD, Kripke DF, Elliott JA, Assmus JD: No association of 6-sulfatoxymelatonin with in-bed $60-\mathrm{Hz}$ magnetic field exposure or illumination level among older adults. Environ Res 2002, 89:201-209.

36. Kennaway DJ, Lushington K, Dawson D, Lack L, Van Den Heuvel C, Rogers $\mathrm{N}$ : Urinary 6-sulfatoxymelatonin excretion and aging: new results and a critical review of the literature. J Pineal Res 1999, 27:210-220.

37. Zhao ZY, Xie Y, Fu YR, Bogdan A, Touitou Y: Aging and the circadian rhythm of melatonin: a cross-sectional study of chinese subjects 30-110 yr of age. Chronobiol Int 2002, 19:1171-1182.

38. Zhou JN, Liu RY, Van Heerikhuize J, Hofman MA, Swaab DF: Alterations in the circadian rhythm of salivary melatonin begin during middle-age. J Pineal Res 2003, 34:11-16.

39. Czeisler CA, Duffy JF, Shanahan TL, Brown EN, Mitchell JF, Rimmer DW, Ronda JM, Silva EJ, Allan JS, Emens JS, Dijk DJ, Kronauer RE: Stability, precision, and near-24-hour period of the human circadian pacemaker. Science 1999, 284:2177-2181.

40. Rea MS, Brons JA, Figueiro MG: Measurements Of Light At Night (Lan) for a sample of female school teachers. Chronobiol Int 2011, 28:673-680.

41. Davis S, Mirick DK, Stevens RG: Night shift work, light at night, and risk of breast cancer. J Natl Cancer Inst 2001, 93:1557-1562.

42. Schernhammer ES, Rosner B, Willett WC, Laden F, Colditz GA, Hankinson SE: Epidemiology of urinary melatonin in women and its relation to other hormones and night work. Cancer Epidemiol Biomarkers Prev 2004, 13:936-943.

43. Bernstein $L$, Allen M, Anton-Culver H, Deapen D, Horn-Ross PL, Peel D, Pinder R, Reynolds P, Sullivan-Halley J, West D, Wright W, Ziogas A, Ross RK: High breast cancer incidence rates among California teachers: results from the California teachers study (United States). Cancer Causes Control 2002, 13:625-635.

44. Gunier RB, Reynolds P, Hurley SE, Yerabati S, Hertz A, Strickland P, Horn-Ross PL: Estimating exposure to polycyclic aromatic hydrocarbons: a comparison of survey, biological monitoring, and geographic information system-based methods. Cancer Epidemiol Biomarkers Prev 2006, 15:1376-1381.

45. Image and Data Processing By Noaa's National Geophysical Data Center: DMSP data collected by the Us Air Force weather agency. http://ngdc noaa.gov/eog/.

46. R Development Core Team: R: A Language And Environment For Statistical Computing. Vienna, Austria: R Foundation For Statistical Computing; 2009.

47. Sun Or Moon Rise/Set Table: 2000. http://aa.usno.navy.mil/data/docs/ RS_OneYear.php.

48. Hartman TJ, Mahabir S, Baer DJ, Stevens RG, Albert PS, Dorgan JF, Kesner JS, Meadows JW, Shields R, Taylor PR: Moderate alcohol consumption and 24-hour urinary levels of melatonin in postmenopausal women. J Clin Endocrinol Metab 2012, 97:E65-E68.

49. Mcpherson M, Janssen I, Grundy A, Tranmer J, Richardson H, Aronson KJ: Physical activity, sedentary behavior, and melatonin among rotating shift nurses. J Occup Environ Med 2011, 53:716-721.

50. Wu AH, Stanczyk FZ, Wang R, Koh WP, Yuan JM, Yu MC: Sleep duration, spot urinary 6-sulfatoxymelatonin levels and risk of breast cancer among chinese women in Singapore. Int J Cancer 2013, 132:891-896.

51. Youngstedt SD, Kripke DF, Elliott JA: Circadian phase-delaying effects of bright light alone and combined with exercise in humans. Am J Physiol Regul Integr Comp Physiol 2002, 282:R259-R266.

52. Lunetta KL, Hayward LB, Segal J, Van Eerdewegh P: Screening large-scale association study data: exploiting interactions using random forests. BMC Genet 2004, 5:32.

doi:10.1186/1476-072X-12-39

Cite this article as: Hurley et al:: A cross-sectional analysis of light at night, neighborhood sociodemographics and urinary 6-sulfatoxymelatonin concentrations: implications for the conduct of health studies. International Journal of Health Geographics 2013 12:39.

\section{Submit your next manuscript to BioMed Central and take full advantage of:}

- Convenient online submission

- Thorough peer review

- No space constraints or color figure charges

- Immediate publication on acceptance

- Inclusion in PubMed, CAS, Scopus and Google Scholar

- Research which is freely available for redistribution

Submit your manuscript at www.biomedcentral.com/submit
C BioMed Central 